\title{
Prognostic Value of Complete Blood Count and Electrolyte Panel during Emergency Department Evaluation for Acute Ischemic Stroke
}

\author{
Latha Ganti, ${ }^{1,2}$ Rachel M. Gilmore, ${ }^{1}$ Amy L. Weaver, ${ }^{1,3}$ and Robert D. Brown Jr. ${ }^{3}$ \\ ${ }^{1}$ Department of Emergency Medicine, Mayo Clinic, Rochester, MN 55905, USA \\ ${ }^{2}$ Department of Emergency Medicine and Neurological Surgery, University of Florida, 1329 SW 16th Street, \\ Suite 4270, Gainesville, FL 32610, USA \\ ${ }^{3}$ Department of Neurology, Mayo Clinic, Rochester, MN 55905, USA
}

Correspondence should be addressed to Latha Ganti; lstead@ufl.edu

Received 19 March 2013; Accepted 11 April 2013

Academic Editors: I. M. Benseñor, A. Ducruet, E. Gonzalez-Toledo, and B. Yulug

Copyright (C) 2013 Latha Ganti et al. This is an open access article distributed under the Creative Commons Attribution License, which permits unrestricted use, distribution, and reproduction in any medium, provided the original work is properly cited.

Objective. To determine whether routine laboratory parameters are predictors of early mortality after acute ischemic stroke (AIS). Methods. The cohort consisted of 522 consecutive patients with AIS presenting to the emergency department (ED) at a tertiary referral center during a 27-month period, residing within the surrounding ten counties. Serum laboratory values were obtained for all patients and categorized according to whether the levels were low, normal, or high. These laboratory results were evaluated as potential predictors of 90-day mortality using Cox proportional hazards models. The associations were summarized by calculating risk ratios (RRs) and 95\% confidence intervals (CI). Results. The presence of elevated white blood cell count (RR 2.2, 95\% CI 1.5-3.4), low bicarbonate (RR 4.2, 95\% CI 2.6-6.7), low calcium (RR 2.9, 95\% CI 1.4-5.9), and high glucose (RR 1.3, 95\% 1.1-1.6) were each univariately associated with significantly higher mortality within the first 90 days. Based on fitting a multivariate Cox regression model, elevated white blood cell count, low bicarbonate, and high glucose were each identified as being jointly associated with early mortality $(P<0.05)$. Conclusion. Early leukocytosis, acidosis, and hyperglycemia and hypocalcemia in AIS appear to be associated with early mortality. Whether addressing these factors will impact survival remains to be investigated.

\section{Introduction}

For patients who present with chief complaint of acute ischemic stroke, the American Stroke Association recommends a set of diagnostic studies [1] to be done at presentation, with the intent of optimizing and expediting the care of these patients. From the Emergency Physicians' perspective many tests are simply part of a routine battery, often without direct impact on emergency department (ED) management, diagnostic or prognostic value. In this study, we sought to determine whether the routine complete blood count (CBC) and electrolyte panel include any components that are markers of early mortality in acute ischemic stroke. Specifically, the parameters of interest were those obtained as part of routine clinical investigation.

\section{Methods}

2.1. Study Design. This study was an observational study using a consecutive sample of local residents presenting to the $\mathrm{ED}$ with acute ischemic stroke (AIS). The primary outcome measure was death at 90 days. This study was approved by the Mayo Clinic Institutional Review Board.

2.2. Study Population and Setting. This study was conducted at the Saint Marys Hospital, a tertiary referral academic medical center with an annual ED census of 70,000 in Rochester, MN. The initial study population consisted of all 723 consecutive patients presenting to the ED with AIS (ICD-9CM codes 433-437) between December 2001 and March 2004. For purposes of follow-up, this sample was limited 
to the 541 patients who resided in the local county and the surrounding 9 county areas. Among these 541, 19 patients denied research authorization and were therefore excluded from further study.

2.3. Study Protocol. The method of patient enrollment was consecutive, and written research authorization was obtained from all patients. All patients regardless of whether they were part of the study or not had standard labs drawn as part of our ED's acute stroke care practice protocol. Our standard practice protocol also includes careful attention to vital sign monitoring, immediate head CT, and neurologic consultation.

2.4. Measurements. Serum laboratory values were obtained in 521 of the 522 patients (one patient was admitted directly to the hospital). The complete blood count (CBC) was performed using the Coulter LH750, an impedance cell counter (Coulter Systems Reference Guide, Beckman-Coulter Corporation, Miami, FL.) The electrolyte panel which includes sodium, potassium, chloride, and bicarbonate was performed using Nova Biomedical M3099859 electrolyte/chemistry analyzer (Nova Biomedical Corp., Waltham, MA). The serum calcium which is frequently collected in addition to standard electrolytes for stroke and cardiac patients was performed using A Copenhagen ABL 700 series Radiometer (Radiometer Medical, Copenhagen, Denmark). Laboratory values were categorized according to whether the levels were low, normal, or high, based on our institution's laboratory reference ranges.

The following information was also abstracted from the medical record: date of birth, gender, date of ED admittance, the date of last follow-up or correspondence, and the date of death. In addition, follow-up was updated at the time of the final data analysis using information from the institutional registration database.

2.5. Data Analysis. The primary outcome variable was mortality at 90 days, as estimated using the Kaplan-Meier method. For patients that died within 90 days, the duration of followup was calculated from the date of the ED admittance to the date of death. The duration of follow-up for all remaining patients was censored at the date of last follow-up if within 90 days or at 91 days. Serum laboratory measurements were considered as potential predictors of early mortality and the categories were evaluated using indicator variables in Cox proportional hazards models, with and without adjusting for gender and age. The associations were summarized by calculating risk ratios (RR) and 95\% confidence intervals (CI). All calculated $P$ values were two-sided and $P$ values less than 0.05 were considered statistically significant. Statistical analyses were performed using the SAS software package (SAS Institute, INC; Cary, North Carolina).

\section{Results}

Among the 521 patients, 276 (53.0\%) were male, 245 (47.0\%) were female, and the mean age at presentation to the ED was 73.1 years (SD 14.5; range 18-101 years). Among the patients known to be alive at last follow-up, the median follow-up was 0.9 years.
A total of 97 patients died within 90 days. The estimated mortality rate ( \pm standard error) at 90 days was $19.4 \% \pm 1.8 \%$. Of the 97 deaths, 34 were within 7 days, 36 were within $8-30$ days, and the remaining 27 were within 31-90 days of the ED visit. The survival within the first 90 days was not significantly different between females and males (21.9\% versus $17.3 \%$; RR 1.3; 95\% CI $0.9-1.9 ; P=0.21$ ). The risk of death within 90 days increased twofold (RR 1.9; 95\% CI 1.5-2.4; $P<0.001$ ) for every increase of 10 years in age.

Based on univariate analyses, the presence of each of the elevated white blood cell count, low bicarbonate, low calcium, low hemoglobin, and high glucose as significantly $(P<0.05)$ associated with mortality within 90 days (Table 1). Similar results were obtained for nearly all of these measures after adjusting for age and gender, with the exception that low hemoglobin was no longer significantly associated with early mortality (Figure 1). Since calcium is not part of the standard electrolyte panel at our institution (only collected in $32.6 \%$ of patients), calcium was not considered in the subsequent analyses. Elevated white blood cell count, low bicarbonate, and high glucose were each identified as being jointly associated with early mortality $(P<0.05)$ based on fitting multivariate Cox regression models and selecting variables for entry based on the likelihood ratio test. Figure 2 illustrates the survival during the first 90 days after presentation to the ED, with the patients categorized according to the presence of high white blood cell count, low bicarbonate, and high glucose levels. Although there were only a total of 14 patients with both low bicarbonate and high glucose, 11 of the 14 died within 90 days after presentation to the ED. These patients are depicted in groups A and B in Figure 2.

\section{Discussion}

The results of our study suggest that amongst the routine labs obtained in the ED evaluation of acute ischemic stroke, an elevated white blood cell count, a low bicarbonate, and a high glucose level are independent predictors of 90-day mortality in the setting of acute ischemic stroke.

4.1. Leukocytosis. The association of leukocytosis has been reported by several investigators. In the Atherosclerosis Risk in Communities (ARIC) Study [2] consisting of 13,555 African-American and White men and women, elevated WBC count was found to be directly associated with increased incidence of coronary heart disease and ischemic stroke. Nadav and colleagues [3] looked at white blood cell count in hospitalized patients and noted it to be an independent risk factor for in-hospital stroke. A subgroup analysis of the Clopidogrel versus Aspirin in Patients at Risk for Ischemic Events (CAPRIE) trial concluded that an increase in leukocyte counts over baseline levels predicts [4] a period of increased risk lasting about one week. This risk was independent of cigarette smoking, one of the factors known to confound elevated $\mathrm{WBC}$ and stroke associations.

The significance of leukocytosis may have to do with the underlying health status of the patient (patients who are sicker to start would be expected to do worse) or may reflect the degree of inflammation triggered by the stroke itself. 
TABLE 1: Association between laboratory measures and early mortality in AIS.

\begin{tabular}{|c|c|c|c|}
\hline Laboratory measure $^{\dagger}$ & $\begin{array}{c}\text { Mortality } \\
\text { at } 90 \text { days } \\
(\%)\end{array}$ & $\begin{array}{c}\text { Risk ratio } \\
(95 \% \text { CI })\end{array}$ & $P$ value \\
\hline \multicolumn{4}{|l|}{ White blood cell count } \\
\hline $\operatorname{Normal}(N=410)$ & 16.4 & Referent & \\
\hline $\operatorname{High}(N=104)$ & 32.8 & $2.2(1.5-3.4)$ & $<0.001$ \\
\hline \multicolumn{4}{|l|}{ Hemoglobin } \\
\hline Low $(N=180)$ & 25.0 & $1.7(1.1-2.5)$ & 0.014 \\
\hline Normal $(N=332)$ & 16.3 & Referent & \\
\hline $\operatorname{High}(N=6)$ & $-^{\ddagger}$ & - & \\
\hline \multicolumn{4}{|l|}{ Platelet count } \\
\hline Low $(N=46)$ & 28.7 & $1.8(1.0-3.2)$ & 0.059 \\
\hline $\operatorname{Normal}(N=461)$ & 18.0 & Referent & \\
\hline $\operatorname{High}(N=10)$ & $-^{\ddagger}$ & - & \\
\hline \multicolumn{4}{|l|}{ Sodium } \\
\hline Low $(N=43)$ & 24.2 & $1.3(0.7-2.6)$ & 0.39 \\
\hline Normal $(N=465)$ & 18.6 & Referent & \\
\hline $\operatorname{High}(N=8)$ & $一^{\ddagger}$ & - & \\
\hline \multicolumn{4}{|l|}{ Potassium } \\
\hline Low $(N=60)$ & 13.6 & $0.7(0.3-1.4)$ & 0.28 \\
\hline Normal $(N=428)$ & 20.6 & Referent & \\
\hline $\operatorname{High}(N=27)$ & 14.8 & $0.7(0.2-1.8)$ & 0.45 \\
\hline \multicolumn{4}{|l|}{ Chloride } \\
\hline Low $(N=100)$ & 18.9 & $1.0(0.6-1.6)$ & 0.88 \\
\hline Normal $(N=381)$ & 18.8 & Referent & \\
\hline $\operatorname{High}(N=34)$ & 29.9 & $1.6(0.8-3.3)$ & 0.17 \\
\hline \multicolumn{4}{|l|}{ Bicarbonate } \\
\hline Low $(N=49)$ & 51.2 & $4.2(2.6-6.7)$ & $<0.001$ \\
\hline Normal $(N=401)$ & 15.6 & Referent & \\
\hline $\operatorname{High}(N=65)$ & 20.1 & $1.3(0.7-2.3)$ & 0.48 \\
\hline \multicolumn{4}{|l|}{ Glucose } \\
\hline Low $(N=3)$ & $-^{\ddagger}$ & - & \\
\hline Normal $(N=363)$ & 17.7 & Referent & \\
\hline $\operatorname{High}(N=118)$ & 27.8 & $1.3(1.1-1.6)$ & 0.010 \\
\hline Not assessed $(N=37)$ & 11.0 & - & \\
\hline \multicolumn{4}{|l|}{ Calcium } \\
\hline Low $(N=48)$ & 33.6 & $2.9(1.4-5.9)$ & 0.003 \\
\hline Normal $(N=116)$ & 13.5 & Referent & \\
\hline $\operatorname{High}(N=6)$ & $-^{\ddagger}$ & - & \\
\hline Not assessed $(N=351)$ & 19.8 & - & \\
\hline
\end{tabular}

${ }^{\dagger}$ Not all patients had every laboratory measure assessed.

₹ There were too few patients with either low glucose or high sodium, calcium, hemoglobin, or platelet count to estimate the mortality at 90 days.

Another etiology may be that of a stress reaction; however, no association with glucose was noted in our study (one would expect to see hyperglycemia in the face of a stress reaction). Although several studies as noted above have shown the association, the exact underlying mechanisms by which

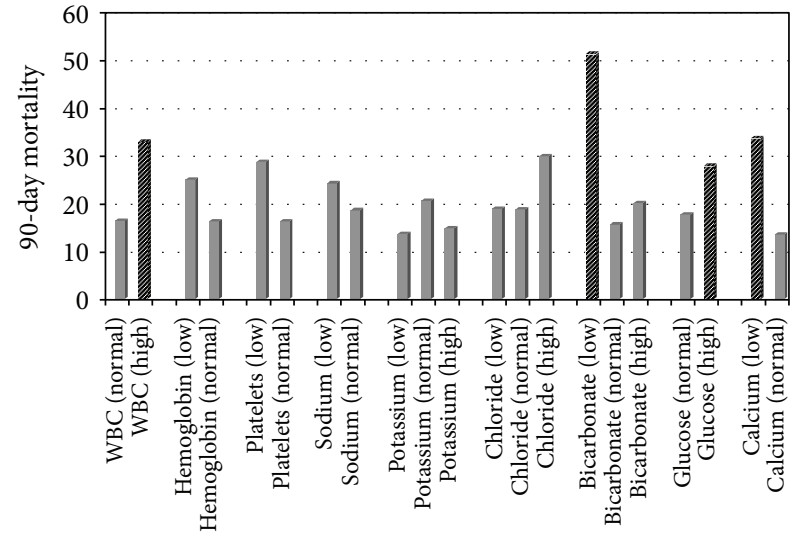

FIgURE 1: Serum laboratory parameters versus 90-day mortality in AIS.

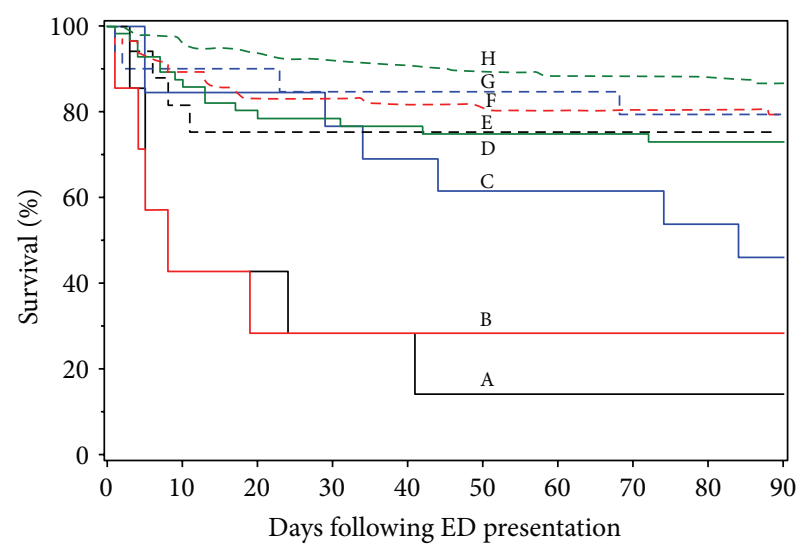

- Low bicarbonate-yes, high WBC-yes, high glucose-yes $(N=7)$

L Low bicarbonate-yes, high WBC-no, high glucose-yes $(N=7)$

Low bicarbonate-yes, high WBC-yes, high glucose-no $(N=13)$

L Low bicarbonate-no, high WBC-yes, high glucose-no $(N=58)$

- - - Low bicarbonate-yes, high WBC-no, high glucose-no $(N=17)$

- - - Low bicarbonate-no, high WBC-no, high glucose-yes $(N=84)$

- - - Low bicarbonate-no, high WBC-yes, high glucose-yes $(N=20)$

- - - Low bicarbonate-no, high WBC-no, high glucose-no $(N=271)$

FIgURE 2: Survival during the first 90 days after presentation to the $\mathrm{ED}$, according to the presence of low bicarbonate, high WBC, and high glucose levels.

leukocyte counts are linked to ischemic risk are unknown at the present time.

4.2. Hyperglycemia. While diabetes is a known risk factor for stroke, hyperglycemia itself appears to be a risk factor for poor prognosis for stroke as well. Gray et al. [5] note an admission glucose of $>145 \mathrm{mg} / \mathrm{dL}$ to be significantly associated with worse mortality at 4 weeks. Weir et al. [6] found hyperglycemia to be predictive of poorer functional outcome at 3 months (alive at home versus in care or dead). Animal models have sought to investigate this association of hyperglycemia with poor stroke outcome further. Experimental hyperglycemia has been induced in cats and shown to increase infarct size [7]. Insulin has been administered in 
an effort to control poststroke hyperglycemia and shown to decrease spatial learning deficit in rats [8]. So far, most of the human studies have focused on retrospective associations of elevated glucose levels (regardless of diabetes) with outcome variables such as death at various time points [9], assessments of functional outcome using Barthel Index [10] and Rankin scales, and placement following hospital discharge (home versus skilled nursing facility). Only recently have trials have begun to assess the logical next step-whether controlling the hyperglycemia in acute ischemic stroke improves outcome [11-13].

The exact mechanism by which hyperglycemia exacerbates acute ischemic stroke outcome is debated. Several theories are postulated, including contribution to vasogenic edema, reduced cerebral blood flow, and increased lactic acid production. Studies have also implicated hyperglycemia in the hemorrhagic transformation of the ischemic infarct [14]. From animal studies, it appears that the most consistent finding is the resulting acidosis produced by hyperglycemia [15]. The next section discusses the possibility of acidosis in acute brain ischemia.

4.3. Acidosis. From the pathophysiology standpoint, the accumulation of lactic acid in the brain milieu can be explained by the ischemic brain's dependence on anaerobic metabolism, which in turn generates lactic acid. Lactate is released as a product of anaerobic metabolism and as such is a nonspecific marker. Saunders et al. [15] demonstrated its presence by short-echo proton spectroscopy in the infarcted brain milieu shortly after focal brain ischemia in humans, and a subsequent fall in lactate levels after the acute infarct had resolved. Markedly elevated cerebral lactate production was also noted in a small cohort of patients with CT proven cerebral infarction following head injury [16].

An interesting extension of this finding of the presence of lactate immediately after acute ischemic stroke is the concept of correcting such a detectable laboratory abnormality. Correction of acidosis in acute stroke was examined by Kuyama and colleagues [17]. They infused an alkalinizing agent, tris(hydroxymethyl) aminomethane, into the perifocal and systemic circulation of cats in whom left MCA occlusion was experimentally achieved. The alkalinization had the effect of reducing cortical edema and infarct size. A similar study was carried out in the rabbit model [18], where again infarct volume was decreased by pretreatment with sodium bicarbonate.

These studies suggest that serum alkalinization may be worthy of investigation as therapy in patients with acute ischemic stroke and low bicarbonate levels. This can be done relatively easily with exogenous bicarbonate, either by the oral or intravenous routes. In terms of safety and feasibility in humans, such therapy is already employed in cases of TCA overdose, bicarbonate responsive acidosis, and severe DKA and as part of chemotherapeutic regimens in human subjects.

4.4. Hypocalcemia. Calcium is an important electrolyte for multiple physiologic processes. For example, it is needed for clotting factors to work, neural transmission, and bone health. Hypocalcemia, which can be caused by PTH or vitamin D deficiency or resistance, dietary deficiency, renal failure, liver failure, and use of certain diuretics (many of these commonplace in the elderly) can result in tetany, seizures, hemorrhagic transformations of infarcts, and osteoporosis. Osteoporosis is a major contributor to falls, hip fractures, and death in the elderly [19]. Hypocalcemia is also known to cause QTc prolongation [20], leading to arrhythmias that may increase severity of stroke. Furthermore, serum hypoglycemia may be reflective of the massive influx of calcium into ischemic penumbra in the brain, which triggers the ischemic cascade to produce free radicals and result in brain infarct. Exactly which of these factors related to hypocalcemia is associated with increased mortality in our study is difficult to speculate on, since only information on mortality, rather than immediate proximal cause of death, was obtained.

\section{Limitations}

There are several important limitations to this study. First, since it was observational in nature, not all patients had all the same labs drawn, especially parameters such as calcium. Second, the initial ED laboratory values may be reflective of the patient's underlying disease, rather than the severity of the stroke itself. Third, patients in our analysis were not stratified by stroke subtype, which again may have confounded the results of the association of labs with mortality. Finally, the parameters noted may simply be independent predictors of mortality, irrespective of any association with acute brain ischemia.

Despite these multiple possible confounders, the previous parameters which are part of routine ED evaluation of the patient with acute ischemic stroke were associated with an increased risk of death at 90 days.

These preliminary results could be further investigated prospectively to see if they can be used as part of a model to predict stroke outcome. Furthermore, the question remains whether correction or manipulation of these observed parameters would result in improved survival.

\section{Conclusions}

The results of our study suggest that amongst the routine labs obtained in the emergency department in the evaluation of acute ischemic stroke, an elevated white blood cell count, a low serum bicarbonate, and a high glucose level are independent predictors of 90-day mortality. Low serum calcium also appears to be associated with worse mortality, although our study design did not permit us to evaluate this result in the multivariate model with the others.

\section{References}

[1] H. P. Adams, R. J. Adams, T. Brott et al., "Guidelines for the early management of patients with ischemic stroke: a scientific statement from the Stroke Council of the American Stroke Association," Stroke, vol. 34, no. 4, pp. 1056-1083, 2003.

[2] C. D. Lee, A. R. Folsom, F. J. Nieto, L. E. Chambless, E. Shahar, and D. A. Wolfe, "White blood cell count and incidence of coronary heart disease and ischemic stroke and mortality 
from cardiovascular disease in African-American and White men and women: atherosclerosis risk in communities study," American Journal of Epidemiology, vol. 154, no. 8, pp. 758-764, 2001.

[3] L. Nadav, A. Y. Gur, A. D. Korczyn, and N. M. Bornstein, "Stroke in hospitalized patients: are there special risk factors?" Cerebrovascular Diseases, vol. 13, no. 2, pp. 127-131, 2002.

[4] A. J. Grau, A. W. Boddy, D. A. Dukovic et al., "Leukocyte count as an independent predictor of recurrent ischemic events," Stroke, vol. 35, no. 5, pp. 1147-1152, 2004.

[5] C. S. Gray, R. Taylor, J. M. French et al., "The prognostic value of stress hyperglycemia and previously unrecognized diabetes mellitus in acute stroke," Diabetic Medicine, vol. 4, no. 3, pp. 237240, 1987.

[6] C. J. Weir, G. D. Murray, K. R. Lees, and A. G. Dyker, "Is hyperglycaemia an independent predictor of poor outcome after acute stroke? Results of a long term follow up study," British Medical Journal, vol. 314, no. 7090, pp. 1303-1306, 1997.

[7] G. de Courten-Myers, R. E. Myers, and L. Schoolfield, "Hyperglycemia enlarges infarct size in cerebrovascular occlusion in cats," Stroke, vol. 19, no. 5, pp. 623-630, 1988.

[8] C. L. Voll, I. Q. Whishaw, and R. N. Auer, "Postischemic insulin reduces spatial learning deficit following transient forebrain ischemia in rats," Stroke, vol. 20, no. 5, pp. 646-651, 1989.

[9] L. S. Williams, J. Rotich, R. Qi et al., "Effects of admission hyperglycemia on mortality and costs in acute ischemic stroke," Neurology, vol. 59, no. 1, pp. 67-71, 2002.

[10] A. Bruno, S. R. Levine, M. R. Frankel et al., "Admission glucose level and clinical outcomes in the NINDS rt-PA stroke trial," Neurology, vol. 59, no. 5, pp. 669-674, 2002.

[11] J. F. Scott, G. M. Robinson, J. M. French, J. E. O'Connell, K. G. M. M. Alberti, and C. S. Gray, "Glucose potassium insulin infusions in the treatment of acute stroke patients with mild to moderate hyperglycemia. The glucose insulin in stroke trial (GIST)," Stroke, vol. 30, no. 4, pp. 793-799, 1999.

[12] C. S. Gray, A. J. Hildreth, J. E. O'Connell, and G. K. Alberti, "The management of post stroke hyperglycaemia (the United Kingdom glucose insulin in stroke trial-GISTUK)," Stroke, vol. 34, no. 1, p. 249, 2003.

[13] T. A. Baird, M. W. Parsons, P. A. Barber et al., "The impact of diabetes and hyperglycaemia on stroke outcome-the GRACE Study," in Proceedings of the Stroke Society of Australasia, p. 18, Auckland, New Zealand, September 2001.

[14] A. M. Demchuk, L. B. Morgenstern, D. W. Krieger et al., "Serum glucose level and diabetes predict tissue plasminogen activatorrelated intracerebral hemorrhage in acute ischemic stroke," Stroke, vol. 30, no. 1, pp. 34-39, 1999.

[15] D. E. Saunders, F. A. Howe, A. van den Boogaart, M. A. McLean, J. R. Griffiths, and M. M. Brown, "Continuing ischemic damage after acute middle cerebral artery infarction in humans demonstrated by short-echo proton spectroscopy," Stroke, vol. 26, no. 6, pp. 1007-1013, 1995.

[16] C. S. Robertson, R. G. Grossman, J. C. Goodman, and R. K. Narayan, "The predictive value of cerebral anaerobic metabolism with cerebral infarction after head injury," Journal of Neurosurgery, vol. 67, no. 3, pp. 361-368, 1987.

[17] H. Kuyama, T. Kitaoka, K. Fujita, and S. Nagao, "The effect of alkalizing agents on experimental focal cerebral ischemia," Acta Neurochirurgica, vol. 60, pp. 325-328, 1994.

[18] R. E. Anderson and F. B. Meyer, "Protection of focal cerebral ischemia by alkalinization of systemic $\mathrm{pH}$," Neurosurgery, vol. 51, no. 5, pp. 1256-1266, 2002.
[19] Y. Sato, H. Kuno, M. Kaji, K. Etoh, and K. Oizumi, "Influence of immobilization upon calcium metabolism in the week following hemiplegic stroke," Journal of the Neurological Sciences, vol. 175, no. 2, pp. 135-139, 2000.

[20] R. Jayasinghe and P. Kovoor, "Drugs and the QTc interval," Australian Prescriber, vol. 25, no. 3, pp. 63-65, 2002. 


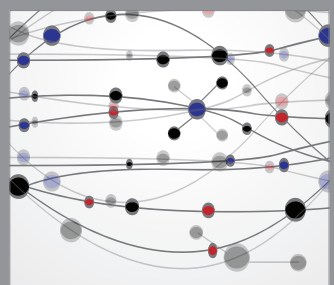

The Scientific World Journal
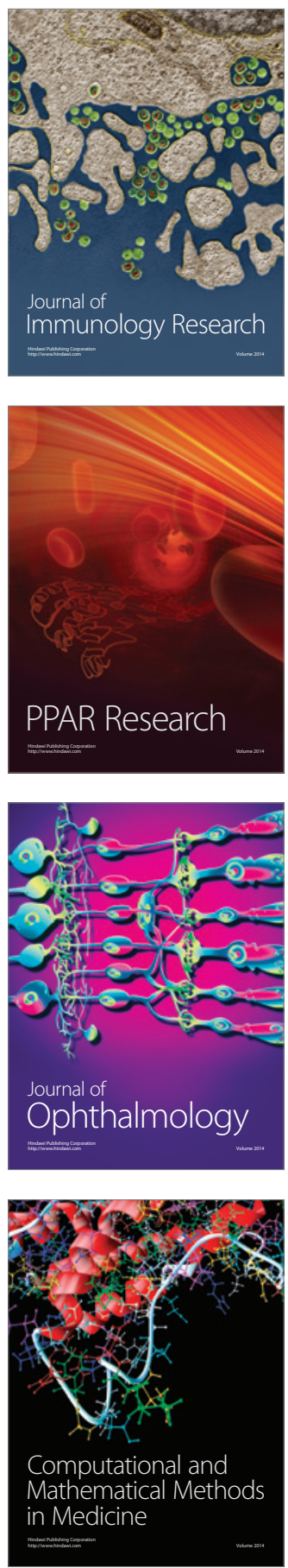

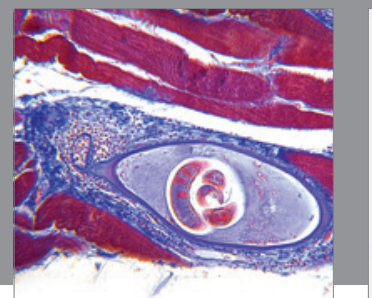

Gastroenterology

Research and Practice
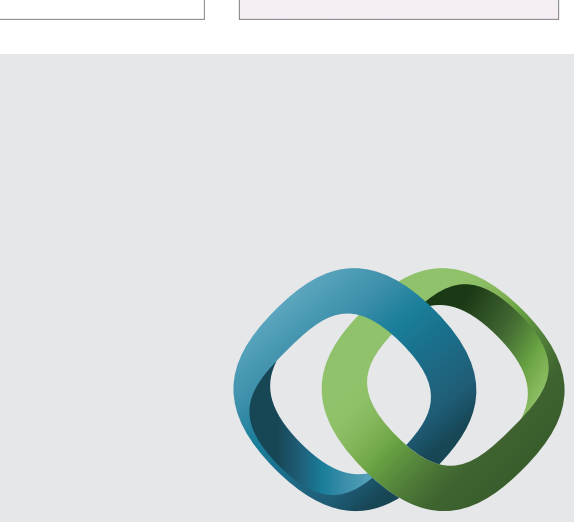

\section{Hindawi}

Submit your manuscripts at

http://www.hindawi.com



Disease Markers
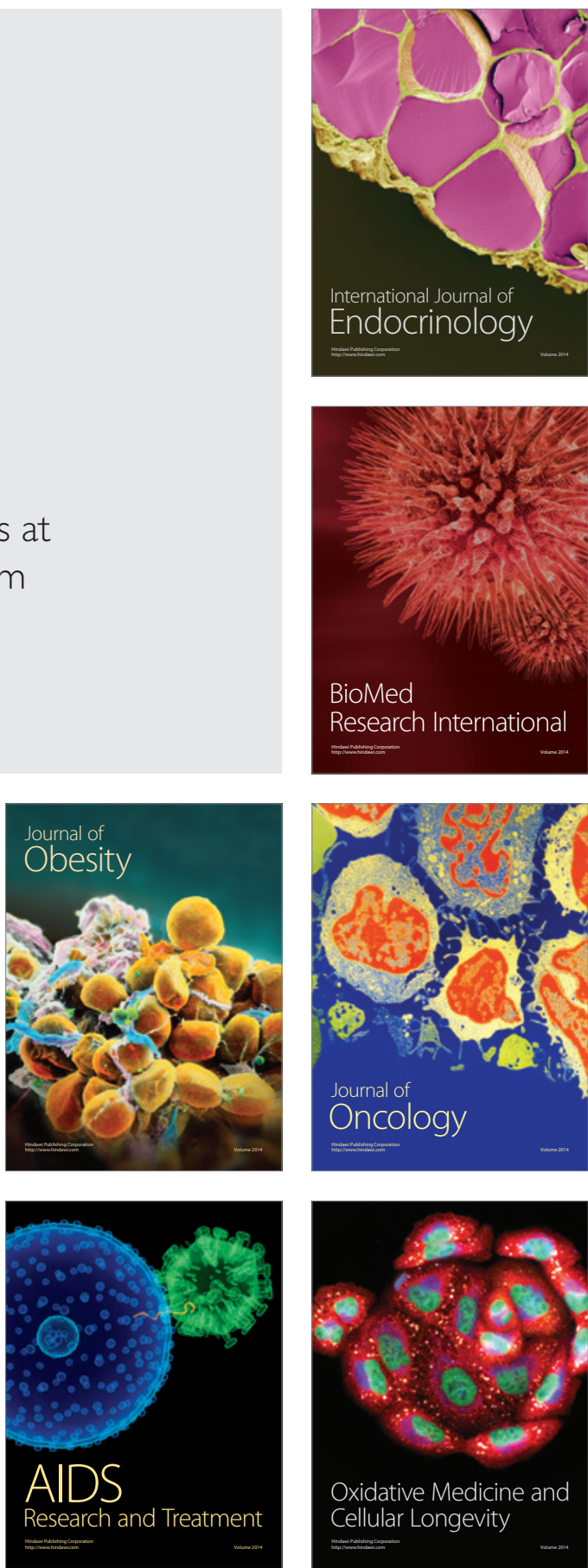